\title{
Von Bibliothekskatalogen zu Wissensportalen
}

\section{Journal Article}

Author(s):

Neubauer, Wolfram (i)

Publication date:

2006-12-19

Permanent link:

https://doi.org/10.3929/ethz-b-000016930

Rights / license:

In Copyright - Non-Commercial Use Permitted

Originally published in:

Bibliothek 30(3), https://doi.org/10.1515/BFUP.2006.275 


\section{Von Bibliothekskatalogen zu Wissensportalen}

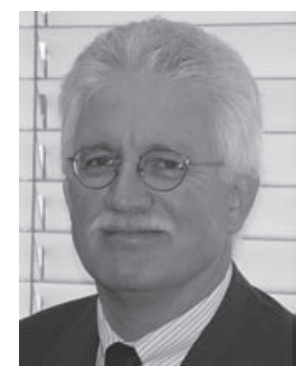

Seit einigen Jahren hat die Diskussion über die Entwicklung und den Aufbau von Bibliotheksportalen erheblich an Bedeutung gewonnen. Auslöser für diesen Prozess ist das zunehmend unübersichtlich werdende elektronische Produktportfolio vieler wissenschaftlicher Bibliotheken. Hierbei reichen die Angebote von den allgegenwärtigen elektronischen Zeitschriften über Datenbanken, audiovisuelle Medien und digitalisiertes Bildmaterial bis hin zu umfassenden Volltextangeboten auf universitätseigenen Dokumentservern.

Das in den letzten Jahren an der ETH Zürich realisierte Projekt myETH stellt nun den Versuch dar, allen Mitgliedern der Universität einen personalisierten (und damit vereinfachten) Zugang auf elektronische Informationsressourcen aller Art zur Verfügung zu stellen.

Die im mittlerweile zweijährigen Echtbetrieb gemachten Erfahrungen zeigen, dass das Portal grundsätzlich akzeptiert wird, die Nutzung der einzelnen Informationspakete allerdings sehr unterschiedlich ist. Vor allem bei den bibliotheksrelevanten Angeboten ist die Akzeptanz bzw. „Marktdurchdringung“ gegenwärtig noch unbefriedigend.

Die Auswertung konkreter Nutzungsergebnisse sowie Interviews mit potentiellen Nutzerinnen und Nutzern bilden die Basis für eine Weiterentwicklung der bibliotheksrelevanten Angebote. Ziel dieser Bemühungen ist es, durch eine enge Verzahnung von Portalanwendung einerseits und neu gestalteter Bibliotheks-Homepage andererseits eine Optimierung des Zugangs zu elektronischen Bibliotheksressourcen zu erreichen.

From library catalogues to knowledge portals

For some years now, the discussion about the creation and development of library portals has become much more important. The cause of this development is the increasingly confused electronic product portfolio of academic libraries. In this connection, what is on offer ranges from the ubiquitous electronic periodicals, via data banks, audiovisual media and digitalised visual material, through to comprehensive ranges of full texts offered on document servers belonging to universities.

The myETH project implemented at the ETH Zürich over the last few years now represents an attempt to make individually-tailored (and therefore simplified) access to all kinds of electronic information resources available to all members of the university.

Experience gained over the course of its now two-year effective operation shows that the portal is accepted in principle, though use of the individual information packages varies widely. In particular in the case of the library-related supply, the level of acceptance or ,market penetration' is still, at present, unsatisfactory.

An analysis of concrete findings regarding use and of interviews with potential users form the basis of a further development of the library-related supply. The aim of these efforts is to optimise access to electronic library resources through a close dovetailing of portal use, on the one hand, and redesigned library home page, on the other.

Des catalogues bibliothécaires aux portails de connaissances

Depuis quelques années, la discussion sur le développement et l'élaboration de portails bibliothécaires a considérablement gagné en importance. Le portefeuille de produits électroniques de plus en plus confus des bibliothèques scientifiques a été le déclencheur de ce développement. A cet égard, les offres vont des journaux électroniques omniprésents, en passant par les banques de données, les médias audiovisuels et le matériel photo numérique, jusqu'aux vastes offres de texte intégral sur les serveurs de documents propres à l'université.

Le projet myETH, mené à bien à l'ETH Zurich ces dernières années, tente l'expérience de mettre un accès personnalisé (et dès lors simplifié) aux sources d'informations électroniques de tous types à la disposition de l'ensemble des membres de l'université.

Les expériences effectivement en cours depuis deux ans maintenant démontrent que le portail est fondamentalement accepté, mais que l'utilisation des différents packages d'informations est toutefois très variée. L'acceptation ou la „pénétration du marché" est actuellement encore insuffisante surtout au niveau des offres pertinentes pour la bibliothèque.

L'évaluation des résultats concrets en termes d'utilisation, de même que des interviews avec des utilisateurs potentiels, constituent le fondement d'un perfectionnement des offres pertinentes pour la bibliothèque. Ces efforts visent à atteindre une optimalisation de l'accès aux ressources bibliothécaires électroniques, grâce à une association étroite entre l'application de portail d'une part, et la page d'accueil remaniée de la bibliothèque d'autre part. 


\section{Was ist im bibliothekarischen Kontext ein Portal?}

Um eine Vorstellung darüber zu bekommen, was unter einem Portal zu verstehen ist, beginnt der Informationsprozess heute mit einer Recherche in der Suchmaschine Google. Allerdings ergibt die Suche mit dem einfachen Begriff „Portal“ nur bei den deutschsprachigen Adressen bereits eine Treffermenge von etwa 150 Millionen Antworten und auch der Suchbegriff „Bibliotheksportal“ führt mit immer noch 65000 Adressen nicht zum Ziel.

Da auch eine Recherche bei Wikipedia nicht wirklich weiterhilft, ist an dieser Stelle ein kurzer Blick auf die Geschichte der Portalidee sicherlich hilfreich.

Der Begriff „Portal“ im bibliothekarischen Sinne ist etwa um die Mitte der 90er Jahre des letzten Jahrhunderts entstanden und dürfte mit dem MyLibrary-Konzept der North Carolina State University ${ }^{1}$ erstmals einem breiteren bibliothekarischen Publikum bekannt geworden sein ${ }^{2}$. Hier wurde also ein Internet-basiertes Bibliotheksangebot entwickelt, dass hinsichtlich der Funktionalitäten letztlich ein mehr oder weniger identisches Abbild kommerzieller Portalanwendungen darstellte. Die Inhalte waren jetzt natürlich bibliothekarischer Natur ${ }^{3}$, so dass sich dieses Bibliotheksportal modellhaft auch als „bibliothekszentriertes Modell“ darstellen lässt. In den Folgejahren kamen dann auch solche Vorstellungen zum Tragen, die einen eher Nutzer- bzw. Kunden-orientierten Ansatz favorisierten, bei dem bibliothekarische Angebote nur einen (häufig kleineren) Teil des Gesamtangebotes an elektronischen Ressourcen bilden. Somit stellen diese beiden Modelle die Eck- bzw. Endpunke bei der Entwicklung bibliothekarischer Portale dar, wobei in der Praxis naturgemäss unterschiedliche Mischformen existieren.

Kommt man nun zur konkreten Frage zurück, was denn eigentlich ein Bibliotheksportal ist und was die wesentlichen Definitionskriterien sein könnten, dann spielen fünf Aspekte eine kritische Rolle:

- Die Strukturierung und Organisation von Informationsquellen

- das Zusammenstellen und Ordnen von Informationsdiensten, Informationsdienstleistungen und WebTools

- die Personalisierung bzw. nutzergruppengerechte Strukturierung des gesamten Portalangebotes in Form von unterschiedlichen Suchumgebungen

- die Integration unterschiedlichster Arten von Daten

- das Schaffen und Pflegen von unterschiedlichen Communities.

Diese relativ weit gefassten Aktionsfelder werden von Dolphin et al. (2002) dann in einer kurzen und prägnanten Definition zusammengefasst: „A (thin) layer which aggregates, integrates, personalises and presents information, transactions and applications to the user according to their role and preferences."

Weitere brauchbare Definitionen bieten einmal das Joint Informations Systems Committee, JISC (Portals 2003) sowie das Fraunhofer Institut für Arbeitswirtschaft und Organisation, IAO (Kirchhof 2004). „Technically, a portal is a network service that brings together content from diverse distributed resources using technologies such as cross searching, harvesting, and alerting, and collate this into an amalgamated form for presentation to the user. This presentation is usually via a web browser, though other means are also possible. For users, a portal is a, possibly personalised, common point of access where searching can be carried out across one or more than one resource and the amalgamated results viewed. Information may also be presented via other means, for example, alerting services and conference listings or links to e-prints and learning materials" (Portals 2003).

„Ein Portal ist definiert als eine Applikation, welche basierend auf Webtechnologien einen zentralen Zugriff auf personalisierte Inhalte sowie bedarfsgerecht auf Prozesse darstellt. Charakterisierend für Portale ist die Verknüpfung und der Datenaustausch zwischen heterogenen Anwendungen über eine Portalplattform. Eine manuelle Anmeldung an den in das Portal integrierten Anwendungen ist durch Single-Sign-On nicht mehr notwendig, es gibt einen zentralen Zugriff über eine homogene Benutzungsoberfläche. Portale bieten die Möglichkeit, Prozesse und Zusammenarbeit innerhalb heterogener Gruppen zu unterstützen" (Kirchhof 2004).

Trotz der offensichtlichen Tatsache, dass die Realisierung von Portalansätzen in jedem Falle sehr viel mit technischen Aspekten zu tun hat, sollte man aus bibliothekarischer Sicht eine wesentliche Randbedingung nicht aus dem Auge verlieren. Im Zentrum aller Bemühungen muss die Frage stehen, wie Bibliothekskunden am einfachsten die für ihre jeweilige Fragestellung relevanten elektronischen Informationen erhalten. Insofern unterscheidet sich diese Situation nicht wesentlich von der, wie sie schon immer die Arbeit von Bibliotheken geprägt hat. Bibliotheksportale sind also lediglich Hilfsmittel für einen möglichst unkomplizierten Zugriff auf das Informationsportfolio von Bibliotheken ${ }^{4}$.

\section{Randbedingungen für die Entwicklung eines Bibliotheksportals an der ETH Zürich}

Wie implizit bereits angesprochen, setzt die Entwicklung und die Einführung eines Bibliotheksportals voraus, dass ein mehr oder weniger umfangreiches elektronisches Informationsangebot vorhanden ist. Um dies zu erreichen, entwickelte die ETH-Bibliothek ${ }^{5}$ etwa ab dem Jahr 1999 ein Konzept, wie und mit welchen Inhalten dies möglich sein könnte. Erklärtes strategisches Ziel bei diesem Kon-

1 North Carolina State University in Raleigh, North Carolina, USA (<http://my.lib.ncsu.edu/>).

2 Die hinter der Realisierung der ersten MyLibrary-Anwendung stehende Philosophie ist im Artikel von E.L. Morgan (2003) gut nachzuvollziehen.

3 Hierzu gehörten: Neuerwerbungslisten, Zugriff zum elektronischen Bibliothekskatalog, Zugriff zu elektronischen Zeitschriften und Datenbanken, Zugriff auf einschlägige WebSeiten. Besonders wichtig war in diesem Beispiel auch der „Zugriff" auf die vielleicht wichtigste Bibliotheksressource, die fachliche Expertise des Bibliothekspersonals.

4 Diese Philosophie stand bereits bei der Entwicklung der ersten bekannten MyLibrary-Anwendung im Mittelpunkt. Vgl. hierzu die Aussagen einer Arbeitsgruppe der North Carolina State University aus dem Jahre 1997 (Morgan 2003).

5 Die ETH-Bibliothek ist die zentrale Informationseinrichtung der ETH Zürich und gleichzeitig die grösste Bibliothek der Schweiz. Details zu elektronischen und Printbeständen finden sich unter: <www.ethbib.ethz.ch/about.html>. 
zept war der möglichst rasche Aufbau einer „Digitalen Bibliothek ETH Zürich“, bei dem die Kernpunkte (vgl. Abb.1) einmal eine nennenswerte Ausweitung des zur Verfügung stehenden Volltextangebotes war und zum Zweiten eine wesentliche Verbesserung der Nachweis- bzw. Zugriffsituation erreicht werden sollte.

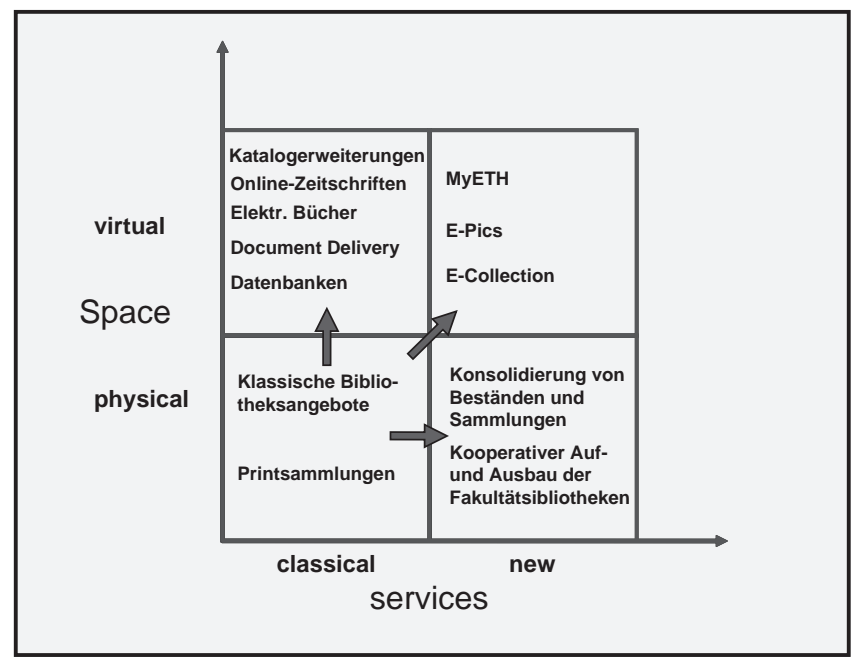

Abb.1: Portfolio elektronischer Informationsangebote der ETH-Bibliothek im Vorfeld der Implementierung von myLibrary (bzw. myETH)

Die Akzeptanz dieser Strategie innerhalb der universitären Communities wurde dann kontinuierlich überprüft, wobei die klassischen Hilfsmittel zum Einsatz kamen (und kommen). Dies sind einmal Befragungen ${ }^{6}$ der unterschiedlichen Zielgruppen und andererseits die kontinuierliche Messung, Auswertung und Interpretation der realen Zugriffe auf unterschiedliche elektronische Angebote.

Die bei diesen Aktivitäten gewonnenen Informationen wurden dann zusammengetragen und bildeten die Basis für die Entwicklung einer konkreten Vision eines Bibliotheksportals, das anfangs unter der Überschrift „myLibrary“ bzw. „myLibrary@ETH“ lanciert wurde. Inhaltlich lässt sich diese Vision mit folgenden wesentlichen Aspekten umreissen:

- Bibliotheksspezifische Inhalte sollten in sachlich oder fachlich strukturierten Informationspaketen bereitgestellt werden, wobei diese aus ganz unterschiedlichen Quellen stammen konnten

- es sollte eine Konfiguration entsprechend Einzel- oder Gruppeninteressen möglich sein (in Form von Semesterapparaten; auf Gruppen- oder Institutsebene; auf Ebene Seminarteilnehmer etc.)

- der Identifizierungsprozess sollte nur einmal notwendig sein (single-sign-on)

- es sollten sowohl universitätsinterne als auch -externe Informationsquellen eingebunden werden können.

Diese jetzt schon einigermassen konkretisierte Projektidee wurde dann durch Interviews bei „fortgeschrittenen Bibliotheksnutzern" hinsichtlich ihrer Brauchbarkeit in der Praxis überprüft, und nachdem die Reaktionen überwiegend positiv ausfielen, fiel die Entscheidung für die Realisierung des Projektes. Da man andererseits davon ausgehen konnte, dass die konkrete Realisierung mit erheblichem Aufwand und Ressourceneinsatz verbunden sein würde, war es sinnvoll, soweit möglich alle technischen und organisatorischen Details in einer Machbarkeitsstudie zu überprüfen. Unter Berücksichtigung internationaler Erfahrungen sollte dabei auch geklärt werden, welcher der unterschiedlichen Realisierungsansätze unter den gegebenen Randbedingungen der sinnvollste war. Die Entscheidung orientierte sich dann sehr stark an den Interessen potentieller Kunden, stellte also die zu erwartenden Ansprüche und Interessen der Kunden in den Mittelpunkt. Somit waren die bibliothekarischen Informationspakete lediglich ein Element in einem grossen Strauss ganz unterschiedlicher Informationsangebote (Abb.2).

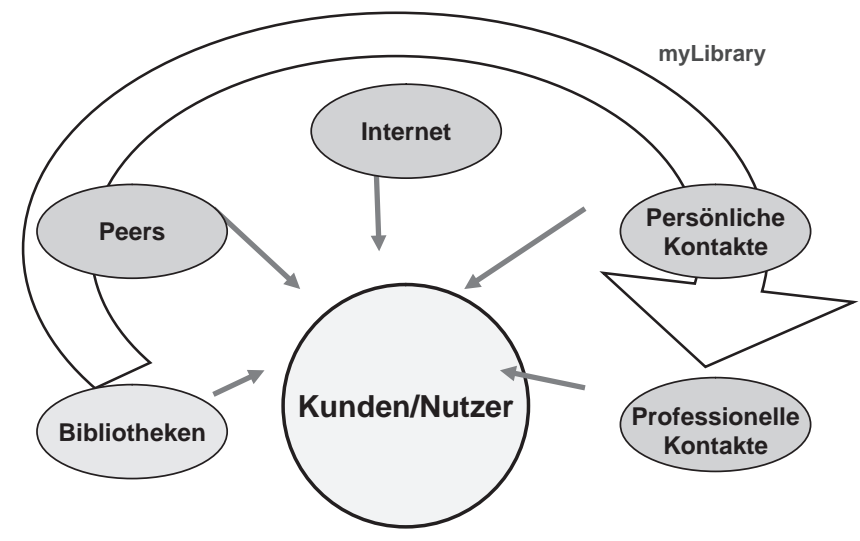

Abb.2: Kundenzentriertes Portalmodell für eine Wissenschaftliche Bibliothek (die bibliothekarischen Inhalte bilden hier nur ein Element eines grösseren, prinzipiell unbegrenzten Informationsangebotes)

\section{3 myLibrary: Die Anwendung}

Die erfolgreiche Realisierung eines Portalprojektes an einer wissenschaftlichen Bibliothek erfordert naturgemäss ausreichende finanzielle und organisatorische Ressourcen, die im Regelfall von einer einzelnen Bibliothekseinrichtung kaum aufgebracht werden können. Im Falle des vorliegenden Projektes war die Situation etwas günstiger, da myLibrary@ETH im Kontext des universitätsweit lancierten Grossprojektes ETH World ${ }^{7}$ verwirklicht werden konnte. Positiv war dies nicht nur aus Gründen einer leichteren Finanzierung, sondern auch aufgrund einer besseren Akzeptanz in den entsprechenden Entscheidungsgremien. Die strategische Initiative ETH World sollte unter anderem dazu beitragen, die gesamte Universität auf die veränderten Bedingungen in den Bereichen wissenschaftliche Information und Kommunikation einzustellen. Eines der wesentlichen strategischen Ziele hierbei war es, eine allgemein zugängliche virtuelle Kommunikations- und Kooperationsplattform zu schaffen. Diese wiederum sollte alle Studierenden und alle Dozierenden bei ihren Aktivitäten an der Universität unterstützen. Somit war es wenig verwunderlich, dass die Entwicklung eines Bibliotheksportals sehr gut in die damalige Projektlandschaft passte.

6 Konkret zum Einsatz kamen mehrere unterschiedliche Methoden: Umfragen bei allen ETH-Angehörigen sowie einzelnen Nutzergruppen vorwiegend in elektronischer Form, Interviews mit ausgewählten „Power-Usern“, Umfragen zu bestimmten Teilportfolios usw.

7 Informationen unter: <www.ethworld.ethz.ch/>. 
Nach einer intensiven bibliotheksinternen Diskussion aller Randbedingungen fiel dann im Jahr 2003 die konkrete Entscheidung, myLibrary@ETH zu realisieren. Das zu diesem Zeitpunkt in einer vorläufigen Version vorliegende Pflichtenheft wurde nochmals überarbeitet, wobei sich die Anstrengungen schwerpunktmässig auf die detaillierte Definition des Produktportfolios innerhalb des Portals konzentrierten. In einer ersten Phase sollte dieses Angebot sechs Informationspakete (sog. channels) umfassen: Dienstleistungen - aktuelle Meldungen - Katalogrecherche - elektronische Zeitschriften/Datenbanken - hochschulinterne und -externe E-Texte - Fachinformationen (Abb.3) ${ }^{8}$.

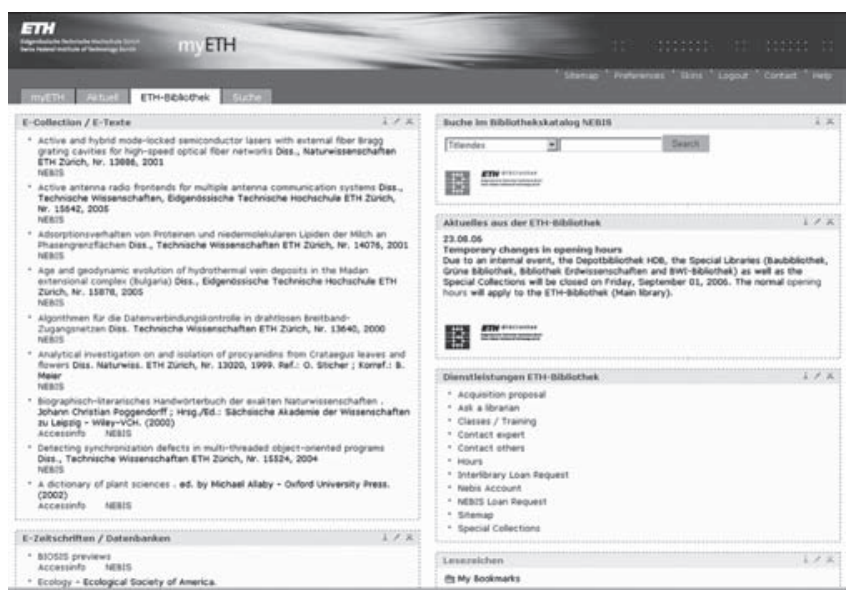

Abb.3: Beispiele für bibliotheksrelevante Informationspakete (= channels) von myETH

Innerhalb dieser Informationspakete können sich nun die Kunden die für sie jeweils relevanten Unterpakete, Fachund Sachgruppen, aber auch einzelne Titel zusammenstellen, um sich auf diese Weise die jeweilige Recherche erheblich zu vereinfachen. Hieraus ergibt sich, dass man nun nicht mehr im gesamten Angebot recherchiert bzw. recherchieren muss, sondern immer nur im einmal definierten Teilportfolio, wobei natürlich Änderungen immer möglich sind.

Die konkrete Umsetzung des myLibrary-Konzeptes bedeutet also aus Benutzersicht, dass man zumindest einmal die Mühe auf sich nehmen und das eigene Informationsbzw. Produktportfolio definieren muss.

Da naturgemäss ein nicht unbeträchtlicher Teil von Bibliothekskunden die Angebote und Dienstleistungen der Bibliothek nur gelegentlich nutzt, dürften diese Personen bei einer Definition ihres Rechercheprofils mehr oder weniger Mühe haben ${ }^{9}$. Um hier Hilfestellung zu geben und auch die Akzeptanz des Portals zu erhöhen, wurden im Rahmen des Projektes sogenannte Default-Seiten entwickelt, die unter Berücksichtigung der individuellen IP-Adresse des Kunden/der Kundin ein vorab definiertes Basisangebot bereitstellen. Konkret bedeutet dies, dass ein Interessent mit einer IP-Identifizierung aus dem Institut für Quantenelektronik der ETH Zürich ein für diesen Bereich charakteristisches Grundangebot an elektronischen Zeitschriftentiteln, Datenbanken, internen und externen Volltexten etc. bekommt und von hier aus sein individuelles Portfolio weiter entwickeln kann.

Auf ein ähnliches Phänomen soll in diesem Zusammenhang ebenfalls hingewiesen werden. Angesprochen ist hier das empirisch gesicherte Faktum, dass ein nicht unbeträchtlicher Teil der Bibliothekskunden zwar die Katalogrecherche für sich selbst besonders interessant einschätzt, gleichzeitig aber Mühe hat, mit dem Rechercheinstrument NEBIS ${ }^{10}$ angemessen umzugehen. Vor allem im Zeitalter von Google und anderen Suchmaschinen sind potentielle und reale Nutzer des Katalogs von der Heterogenität der Recherchemöglichkeiten eher überfordert und wünschen sich aus diesem Grunde eine „Suche ohne grossen Aufwand".

Aus dieser Situation heraus entwickelte das Projektteam von myLibrary in Kooperation mit Bibliotheksspezialistinnen sogenannte „short cuts“ innerhalb des Channels „Katalogrecherche“. Diese sind als priorisierte Suchfunktion definiert (also etwa „Autor“, „Stichwort" oder „Titel“) und reichen die entsprechende Recherche ohne Umwege an den Bibliothekskatalog weiter. Die mehrstufige Identifizierungs- und Einwahlprozedur entfällt auf diese Weise.

\section{Das Kooperationsprojekt myETH}

Wie erwähnt, wurde myLibrary@ETH als Teilprojekt des gesamtuniversitären Grossprojektes ETH World initiiert und umgesetzt, wobei an dieser Stelle erwähnt werden sollte, dass im Rahmen von ETH World auch ein weiteres Portalprojekt ${ }^{11}$ unterstützt wurde. In diesem Falle ging es ebenfalls um ein ETH-weites Portal, das im Rahmen der Feiern zum 150-jährigen Gründungsjubiläum der ETH Zürich in Angriff genommen worden war. Obwohl dieses Projekt sich explizit nicht auf bibliothekarische Inhalte fokussierte und eher Marketingaspekte verfolgte, waren sich die beiden Projekte hinsichtlich Zielsetzung und Realisierungsansatz doch relativ ähnlich.

Somit ist es nicht überraschend, dass nach ersten Diskussionen der jeweiligen Projektverantwortlichen relativ rasch die Idee auftauchte, die beiden Projekte zu vereinigen, da eben die grundsätzlichen Zielsetzungen, aber auch die anvisierten technischen Lösungsansätze in hohem Masse identisch waren. Die Tatsache, dass sich bei einer kooperativen Lösung sehr wahrscheinlich organisatorische und finanzielle Synergien ergeben würden, unterstützte eine Entscheidung in Richtung Zusammenarbeit

8 Die in dieser Darstellung von myETH aufgelisteten Channels waren natürlich bereits Teil von myLibrary.

9 Diese Aussage konnte im späteren Routinebetrieb eindeutig bestätigt werden. Vor allem das angebotene Definitionsprocedere war nicht für alle Interessenten selbst erklärend, woraus sich ein nicht unerheblicher Schulungsaufwand ergeben hat. Grundsätzlich dürfte von einer einfachen Recherchelogik, aber auch von einer nutzerorientiert gestalteten Rechercheoberfläche die Akzeptanz des Portals ganz wesentlich abhängen.

${ }^{10}$ NEBIS ist die Bezeichnung für den Online-Katalog der ETH Zürich, in dem alle Bestände der ETH Zürich, der Zentralbibliothek Zürich, der ETH Lausanne und etwa 80 weiterer Bibliotheken innerhalb der Schweiz nachgewiesen sind. Als Software wird das Produkt Aleph500 der Fa. ExLibris eingesetzt.

11 Das „ETH-Portal“ wurde zu diesem Zeitpunkt von den beiden ETH-Einrichtungen „Corporate Communications“ (= Öffentlichkeitsarbeit) und (zentrale) Informatikdienste (entspricht in etwa den Rechenzentren deutscher Universitäten) entwickelt. 
ebenso, wie die Tatsache, dass es nur schwer möglich gewesen wäre, den Angehörigen der ETH Zürich die Nutzung von zwei doch sehr ähnlichen Portalanwendungen zu vermitteln.

Ebenfalls angesprochen werden sollten an dieser Stelle zwei weitere Aspekte, die für die Wahrnehmung der Bibliothek innerhalb der Universität nicht zu unterschätzen sind. Durch die Realisierung eines in hohem Masse technisch ausgerichteten, die ganze Universität umfassenden Projektes kann sich die Bibliothek über ihre klassische Rolle hinaus auch als wichtiger und vor allem kompetenter Partner bei der Implementierung kundenorientierter, elektronischer Informationsangebote positionieren. Darüber hinaus wurde zum damaligen Zeitpunkt deutlich, dass ein universitätsweites Portal ohne bibliothekarische Inhalte mehr oder weniger keine im engeren Sinne wissenschaftsorientierten Inhalte anbieten kann ${ }^{12}$.

Die konkrete Integration der beiden Portalprojekte myLibra-

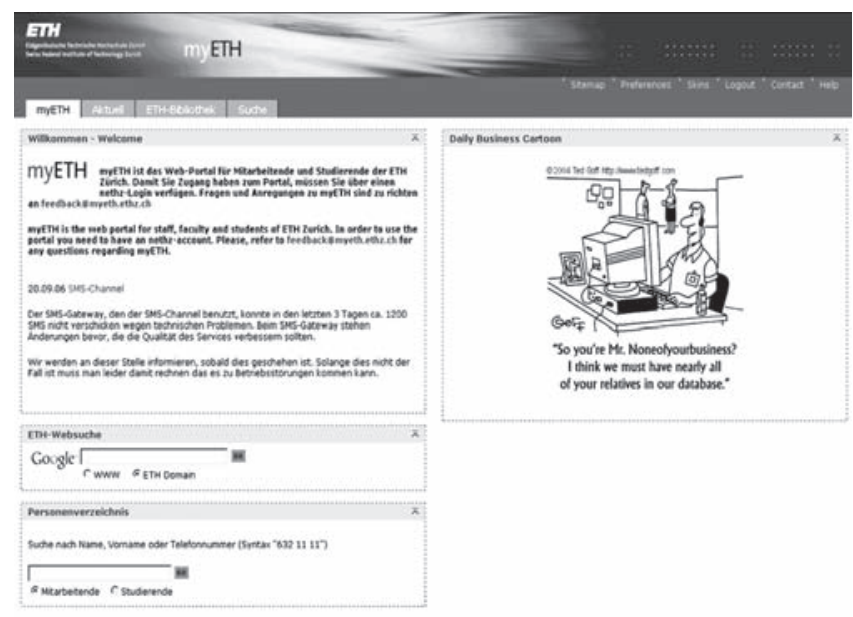

Abb.4: Gegenwärtige Einstiegsseite des Portals myETH

ry und ETH-Portal zum neuen Universitätsportal myETH (Abb.4) begann Anfang des Jahres 2004, wodurch allerdings die reale Nutzung der bibliothekarischen Inhalte durch die ETH-Community nicht unerheblich verzögert wurde. Schliesslich mussten ja aus Sicht der Bibliothek "fremde“ Inhalte integriert werden, und gleichzeitig sollten sich sehr unterschiedliche Arbeitsstrategien zusammenfinden.

Darüber hinaus ergaben sich aus der Fusion der Projekte erhebliche technische und organisatorische Konsequenzen, da beispielsweise die für myLibrary bereits weitgehend aufgebaute Rechner-Infrastruktur durch neue Systeme ersetzt werden musste. Auch wurde es notwendig, bereits entwickelte Programmteile der beiden Softwareapplikationen umzuarbeiten und zu vereinheitlichen.

Zu den technischen Aspekten von myETH sollen an dieser Stelle nur einige wenige Anmerkungen genügen.

Sowohl myLibrary als auch die weiteren Portalaktivitäten an der ETH Zürich stützten bzw. stützen sich hinsichtlich ihrer Softwareplattform auf die Open Source-Anwendung uPortal ${ }^{13}$, die im Hochschulbereich weltweit sehr verbreitet ist. Da es sich bei dieser Software letztlich um einen Werkzeugkasten und nicht um ein fertiges Produkt handelt, mussten im Rahmen der Projekte myETH bzw. myLibrary eine ganze Reihe von Weiterentwicklungen und Ergänzungen realisiert werden, die dann erst zu einer funktionierenden Portalanwendung geformt werden konnten. Wie bereits angesprochen, bilden die Channels (Abb.5) mehr oder weniger das Zentrum von myETH und beanspruchten aus diesem Grund auch den Schwerpunkt der Aufmerksamkeit. Die in XML aufbereiteten Daten aus dem Bibliothekssystem oder anderen Quellen werden in verschiedenen Datenbanken abgelegt und über eine sog. „Channel-Datenbank" verwaltet. Hier werden sowohl die persönlichen Einstellungen der Nutzerinnen und Nutzer überwacht als auch die Recherchen durchgeführt.

Nach dem Durchlaufen des Einwahlprozesses wird über eine sog. LDAP-Schnittstelle in der zentralen Nutzerdatenbank die Zugangsberechtigung geprüft und hierzu parallel abgeklärt, ob für den jeweiligen Nutzer bereits ein persönlicher Portalzugang existiert.

\begin{tabular}{|l|}
\hline$\square_{\text {Aktuelles aus der ETH-Bibliothek }}$ \\
$\square_{\text {Daily Business Cartoon }}$ \\
$\square_{\text {Das Weiterbildungsangebot der ETH }}$ \\
$\square_{\text {Dienstleistungen ETH-Bibliothek }}$ \\
$\square_{\text {E-Collection / E-Texte }}$ \\
$\square_{\text {ETH Index }}$ \\
$\square_{\text {ETH Life }}$ \\
$\square_{\text {ETH Veranstaltungen }}$ \\
$\square_{\text {ETH-Websuche }}$ \\
$\square_{\text {E-Zeitschriften / Datenbanken }}$ \\
$\square_{\text {Gast-Info }}$ \\
$\square_{\text {Intern }}$ \\
$\square_{\text {ISN }}$ \\
$\square_{\text {Kalender }}$ \\
\hline
\end{tabular}

\begin{tabular}{|l|}
\hline Lesezeichen \\
$\square_{\text {Linktool }}$ \\
$\square_{\text {Mensa }}$ \\
$\square_{\text {myETH News welcome page }}$ \\
$\square_{\text {offene Stellen an der ETH }}$ \\
$\square_{\text {Personenverzeichnis }}$ \\
$\square_{\text {Radio \& TV }}$ \\
$\square_{\text {RSS-Reader }}$ \\
$\square_{\text {Suche im Bibliothekskatalog NEBIS }}$ \\
$\square_{\text {VPP-Channel (Verteiltes Printen und Plotten) }}$ \\
$\square_{\text {Web to SMS }}$ \\
$\square_{\text {Willkommen - Welcome }}$ \\
\hline
\end{tabular}

Abb.5: Gesamtangebot an Informationspaketen von myETH (Stand: August 2006)

\section{$5 \quad$ Nutzungsaspekte}

Naturgemäss ist die Nutzung einer Bibliotheksdienstleistung das wesentliche Kriterium bei der Beantwortung der Frage, ob die Investitionen in Sach- und Personalmittel auch gerechtfertigt sind. Ein wesentliches Element bei der Beantwortung dieser Frage ist der Aspekt der Akzeptanz durch die primären Zielgruppen, nach der „Marktdurchdringung", also nach der Nutzung durch die Mitglieder der Universität.

Im vorliegenden Beispiel lässt sich diese Frage grundsätzlich positiv beantworten, wenn man davon ausgeht, dass sich die Zahl der universitären Nutzer von etwa 1880 im Monat Juni 2004 auf etwa 14000 im gleichen Monat des Jahres 2006 erhöht hat ${ }^{14}$. Hieraus lässt sich schliessen, dass etwa zwei Drittel der Universitäts-Community das Portal zumindest grundsätzlich akzeptieren und in irgendeiner Form nutzen. Was für die Nutzerinnen

12 Obwohl diese Aussage auch heute noch weitgehend der Wirklichkeit entspricht, bedeutet dies andererseits jedoch nicht, dass die Akzeptanz bzw. Nutzung der bibliothekarischen Inhalte dadurch besonders erhöht wäre (vgl. Abschn. 5).

${ }^{13}$ Gegenwärtig ist die Version 2.2 im Einsatz. Nähere Informationen zu dieser Software und allen damit in Verbindung stehenden Aspekten findet man unter: <http://uportal.org/>.

14 Im Sommer 2006 waren an der ETH Zürich insgesamt etwa 20000 Personen „beschäftigt"; in dieser Zahl sind die Studierenden inbegriffen. 
und Nutzer innerhalb des Portalangebots im Detail interessant ist, macht Abbildung 6 sehr deutlich. Mit grossem Abstand das beliebteste Angebot ist der "SMS-Channel“, also die Möglichkeit, bis zu 10 SMS/pro Tag unentgeltlich zu verschicken. Mit den elektronischen Zeitschriften bzw. Datenbanken folgt die erste bibliothekarische Anwendung erst im Mittelfeld. Diese Situation korrespondiert dann sehr

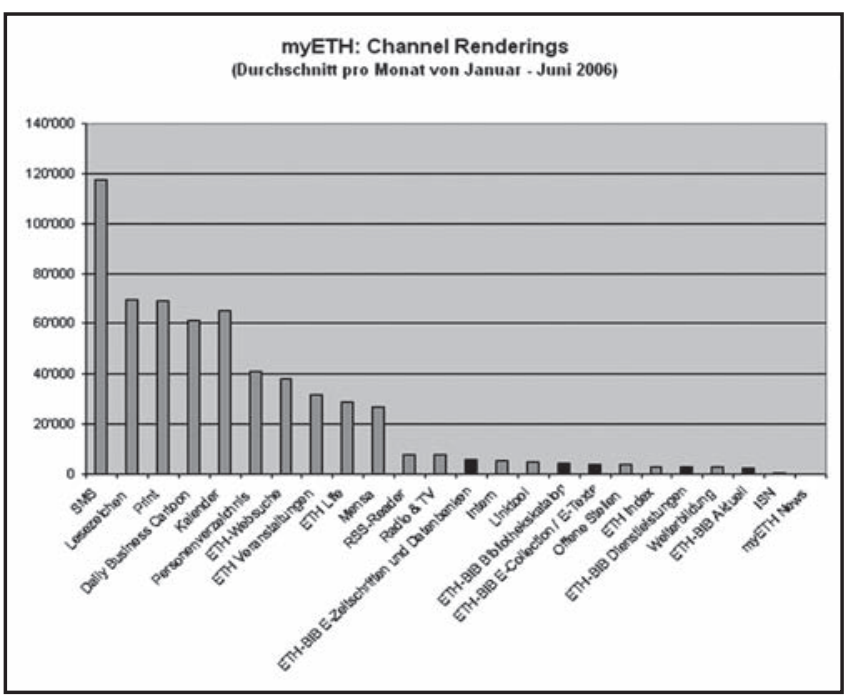

Abb.6: Nutzung der einzelnen Channels von MyETH (Recherchen/Monat; Januar-Juni 2006)

klar mit der Antwort auf die Frage, wer denn die Nutzer/ Nutzerinnen von myETH sind? Die Darstellung in Abbildung 7 zeigt auf, dass die Nutzung der einzelnen Channels eindeutig mit der mengenmässigen Verteilung der einzelnen Nutzergruppen korreliert.

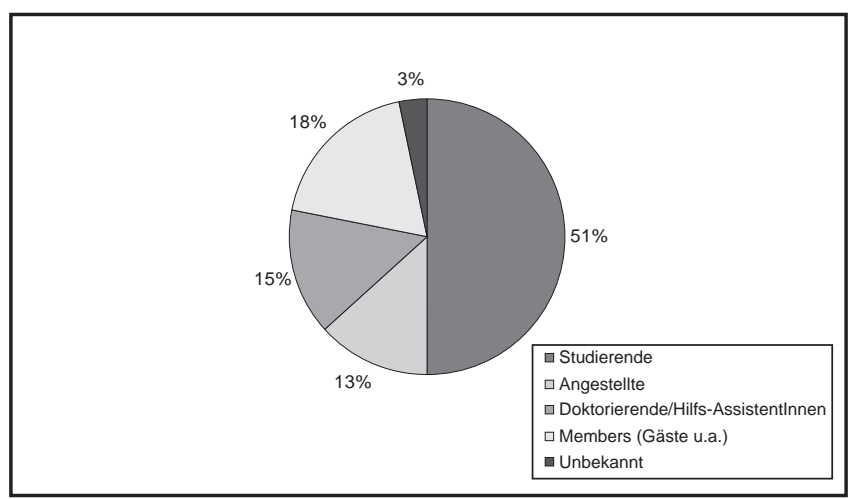

Abb.7: Nutzung von myETH nach Nutzergruppen ${ }^{15}$ (Angaben in \% der Gesamtnutzung; Stand: April 2006)

Bei der Interpretation der Zahlen wird deutlich, dass das Portal von vielen Mitgliedern der ETH Zürich (auch intensiv) genutzt wird, dass die Nutzung der einzelnen Channels allerdings sehr unterschiedlich ist und sich gleichzeitig stark an den Interessen der Studierenden orientiert. Dies ist selbstverständlich nicht negativ zu werten, bedeutet dies doch immerhin, dass eine sehr wichtige Zielgruppe aller Wahrscheinlichkeit nach mit dem Angebot zufrieden ist ${ }^{16}$.

Aus bibliothekarischer Sicht stellt sich die Situation allerdings etwas anders dar. Betrachtet man die Positionierung der bibliothekarischen Angebote im Gesamtportfo- lio, ist leicht erkennbar, dass die Nutzung und damit die Akzeptanz unbefriedigend ist. Der meistgenutzte Bibliotheks-Channel weist deutlich weniger als 10000 Recherchen/Monat auf und liegt damit mehr als den Faktor 10 niedriger als der Spitzenreiter.

Hiermit wird deutlich, dass die absolute Zahl der Zugriffe auf die bibliothekarischen Informationspakete nicht den Erwartungen entspricht, wobei hierzu parallel zu erkennen ist, dass die generelle Nutzung bibliothekarischer Informationsangebote an der ETH Zürich andererseits hoch bis sehr hoch ist. Verdeutlicht wird dies durch die Darstellung in Abbildung 8. Anhand der Zugriffszahlen auf den Channel „Aktuelles aus der ETH-Bibliothek" lässt sich zeigen, dass das Angebot prinzipiell sehr wohl Interesse findet, dass allerdings der Zugangsweg myETH nicht die erste Priorität geniesst. Noch deutlicher zeigt sich das Problem des Zugriffsweges bei den Channels „E-collection" und "Bibliothekskatalog NEBIS". Im ersteren Falle stehen zwölf Zugriffen über myETH mehr als 80 000(!) Zugriffe über die Homepage gegenüber. Am Beispiel des Bibliothekskataloges wird dann endgültig klar, dass ein erheblicher Teil potentieller Nutzerinnen und Nutzer der Bibliotheksangebote myETH nicht als primären Rechercheweg betrachtet ${ }^{17}$.

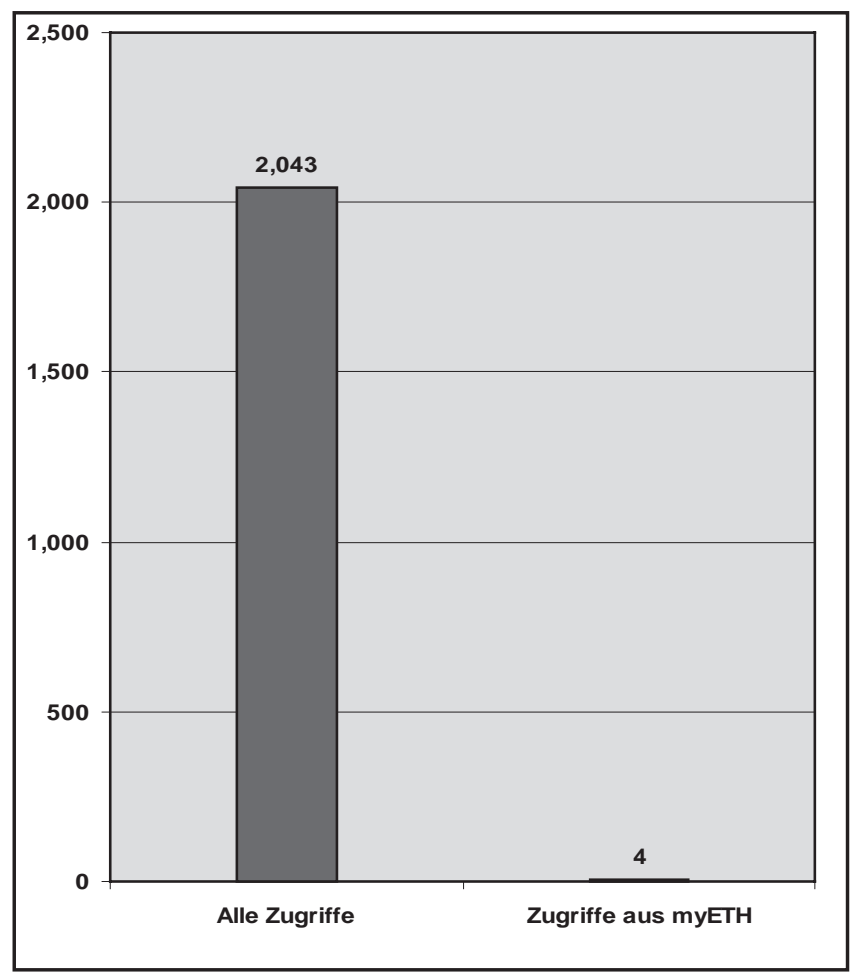

Abb.8: Vergleich der Zugriffszahlen auf den Channel „Aktuelles aus der ETH-Bilbiothek

15 Unter Members sind „Nicht-ETH-Angehörige“ zu verstehen, also beispielsweise Gäste, exmatrikulierte Studierende bis sechs Monate nach ihrem Ausscheiden, kurzfristige Mitarbeitende.

${ }^{16}$ Diese Zufriedenheit wurde auch durch einige Online-Umfragen bestätigt.

17 Im ersten Halbjahr 2006 standen durchschnittlich 96(!) monatlichen Recherchen im Bibliothekskatalog mehrere Millionen Recherchen über die Homepage gegenüber. Obwohl die Zählmethode nicht eindeutige Zahlen ergibt, sind die Grössenordnungen jedoch eindeutig. 
Bei einer Bewertung der praktischen Erfahrungen aus den ersten beiden Betriebsjahren lassen sich somit aus bibliothekarischer Sicht einige wichtige Erkenntnisse ableiten.

- Die Bibliothekskunden finden über das Portal myETH offensichtlich zu wenige Anreize für eine Nutzung der für sie prinzipiell interessanten Angebote

- die Bedienungsfreundlichkeit und das Layout des Gesamtangebotes ist offensichtlich verbesserungsfähig

- die bibliothekarischen Inhalte und die Zielgruppen des Gesamtportals sind nicht optimal aufeinander abgestimmt

- die technische Plattform ist möglicherweise nicht optimal konfiguriert.

Die bibliotheksinternen Diskussionen über die Nutzerakzeptanz bibliothekarischer Informationspakete hat nun vor allem in den letzten Monaten dazu geführt, die grundsätzliche Idee des Portals myETH unter Berücksichtigung der veränderten Randbedingungen neu zu diskutieren. Im Zentrum dieser Überlegungen steht dabei die Frage, wie es gelingen kann, die äusserst umfassenden elektronischen Bibliotheksangebote einem sehr viel grösseren Nutzerkreis zu öffnen. Hierbei ist klar, dass dies erfolgreich nur dann geschehen kann, wenn es gelingt, die Zugangs- und Definitionsprozesse so einfach als möglich zu gestalten. Letztendlich sind alle Bibliothekskunden nur daran interessiert, unkompliziert und rasch zu den für sie relevanten Informationen zu kommen.

\section{$6 \quad$ Was ist mit der Zukunft?}

Welche realen Möglichkeiten gibt es nun, die bibliothekarischen Anteile von myETH besser und gleichzeitig kundenfreundlicher zu präsentieren?

Aus Sicht der ETH-Bibliothek bestehen hier zwei grundsätzliche Möglichkeiten. Ein Ansatz könnte sein, das vorhandene Angebot zu optimieren. Dies würde bedeuten: eine Verbesserung des Zugangs, eine Weiterentwicklung des Layouts sowie eine deutliche Intensivierung entsprechender Marketingmassnahmen.

Nicht zu beheben wäre durch diese Aktivitäten allerdings die Situation, dass die bibliothekarischen Channels nur einen Teil des Gesamtportfolios darstellen und somit immer die Gefahr besteht, dass die Bibliotheksangebote nicht angemessen wahrgenommen werden ${ }^{18}$. Die zweite Möglichkeit ist eine Abkehr vom gewählten „nutzerorientierten Modell“ und gewissermassen eine Rückbesinnung auf den „bibliothekszentrierten Ansatz“.

Nach längeren bibliotheksinternen Diskussionen hat sich die Bibliothek dafür entschieden, zumindest vom Grundsatz her auf den letzteren Ansatz zurückzukommen, dies allerdings mit einer kompletten Neugestaltung des WebAuftritts der Bibliothek zu verbinden. Hierbei verfolgt die ETH-Bibliothek folgende allgemeine Ziele ${ }^{19}$ :

- Rückorientierung auf schwerpunktmässig bibliothekarische Inhalte

- Integration von Bibliotheks-Homepage und myLibraryZugriff

- ETH-interne und -externe Nutzer recherchieren über eine Rechercheoberfläche

- Darstellung der relevanten Inhalte bereits auf der Präsentationsebene

- Metasuche über das gesamte heterogene Produktportfolio
- für interne Nutzer ist nur ein Login notwendig

- Differenzierung der Rechercheergebnisse nach Fächern und/oder nach Medientypen

- das Angebot ist eindeutig als Bibliotheksdienstleistung erkennbar

- möglichst stringente Realisierung des Projektes (bis Ende 2007).

Neues strategisches Ziel ist somit der Aufbau eines „Wissensportal ETH Zürich", also die Schaffung eines zentralen und gleichzeitig integrierten Zugangssystems für die an der ETH-Bibliothek vorhandenen bzw. zugreifbaren heterogenen Informationsquellen, elektronischen Ressourcen, Printformate sowie für das umfassende Angebot an Informationsdienstleistungen.

Es geht also auch hier um die Schaffung eines Portals, eines „Single point of access" für die übergreifende Recherche und Bereitstellung von Dokumenten aller Medientypen.

Der Aktualitätswert dieses Wissensportals liegt allerdings primär nicht darin, dass sich dort bisher völlig unbekannte Medientypen oder Informationsdienstleistungen finden würden. Der aus Nutzersicht interessante Aspekt ist die Integration von „üblicher" Homepage einer wissenschaftlichen Grossbibliothek einerseits mit den spezifischen Besonderheiten eines Bibliotheksportals andererseits. Die ETH-Bibliothek verspricht sich hiervon eine deutlich bessere Nutzung des elektronischen Produktportfolios sowie eine erkennbar bessere "Marktdurchdringung".

\section{Schlussfolgerungen}

Im Kontext der skizzierten Erfahrungen an der ETH Zürich zeigt sich, dass die theoretisch postulierten Vorteile der Integration bibliothekarischer Portalanwendungen in inhaltlich weiter gefasste Universitätsportale in der Praxis des bibliothekarischen Alltags nicht ohne weiteres realisiert werden können. Bei einer detaillierten Ursachenforschung sollte man sich deshalb immer bewusst sein, dass eine deutliche Ausweitung des Informationsportfolios für Nutzerinnen und Nutzer einer Bibliothek immer auch eine erweiterte Komplexität bei der Recherche bedeutet, die durch Kontextualisierung oder den Einsatz von nutzerfokussierten myLibrary-Awendungen zwar verringert, aber nicht aufgelöst werden kann.

Im Falle des vorliegenden Beispiels liegt die Vermutung nahe, dass mit den hier bereitgestellten Rechercheinstrumenten einerseits die jeweilige Einzelinformation in der überwältigenden Menge an potentiell relevanter Information nicht mehr erkennbar war. Andererseits könnten die konkreten Erfahrungen auch dahingehend interpretiert werden, dass die bereitgestellte Menge an Informationspaketen und deren inhaltliche Heterogenität die für bibliothekarische Anwendungen eigentlich notwendige Definition der Zielgruppen nicht mehr in ausreichendem Masse möglich machte. Mit anderen Worten: Sind die Zielgruppen nicht relativ eng definiert, ist das für jede dieser Gruppen potentiell relevante Angebot immer noch so gross, dass

${ }^{18}$ Wie skizziert, ist diese Situation auch die gegenwärtige Realität.

19 In den Abbildungen 9-12 sind mögliche Präsentationsformen für diese Variante in exemplarischer Form dargestellt. 
es vom Einzelnen nicht überblickt werden kann. Weiter ergibt sich aus diesem Gedankengang, dass eine wirklich detaillierte Definition von bibliothekarischen Zielgruppen in erheblichem Umfang Ressourcen für deren Generierung und Pflege erfordern würde. Es ist kaum vorstellbar, dass dies von einer einzelnen Bibliothek überhaupt noch geleistet werden kann.

Die im vorliegenden Beispiel vorgesehene Integration von Portalanwendungen und neu strukturierter Homepage der Bibliothek als einzigen Einstieg in das elektronische Informationsangebot ist somit eine Weiterentwicklung des Portalgedankens, die in gewisser Weise mit einer Rückbesinnung auf stärker bibliothekarische Inhalte Hand in Hand geht. Dies bedeutet dann naturgemäss (zumindest bis zu einem gewissen Masse) eine Abkehr von der Vision, dass bibliothekarische Fachportale zu nationalen oder sogar internationalen „Drehscheiben für den Wissenstransfer" (Guba 2006) werden könnten.

\section{$8 \quad$ Literatur}

Ciccone, Karen: MyLibrary@NCState: A library portal after five years. In: Journal of Library Administration 43 (2005) No.1/2, pp.19-35.

Cox, Andrew [Hrsg.]: Portals. People, processes and technology. London 2006.

Dolphin, I.; Miller, P. und R. Sherratt: Portals, portals everywhere. In: Ariadne 33 (2002) (<www.ariadne.ac.uk/ issue33/portals>)

Guba, Beate: MyDUK. Vom Nutzen eines Informationsund Kommunikationsportals im Fall der Donau-Universität Krems. Krems 2006.

Guba, Beate: Bibliotheken und Portale - Ein Überblick. In: GMS Medizin-Bibliothek-Information 6 (2006) Nr. 2.

Jutzi, Ursula: MyLibrary@ETH: Ziel - Konzept - Realisierung. Vortrag gehalten an der TIB/UB Hannover am 28.08. 2003 (<www.ethbib.ethz.ch/pub/my_library_tib. $\mathrm{ppt}>$ ).

Kirchhof, Anja; Gurzki, Thorsten; Hinderer, Henning und Joannis Vlachakis: Was ist ein Portal? Definition und

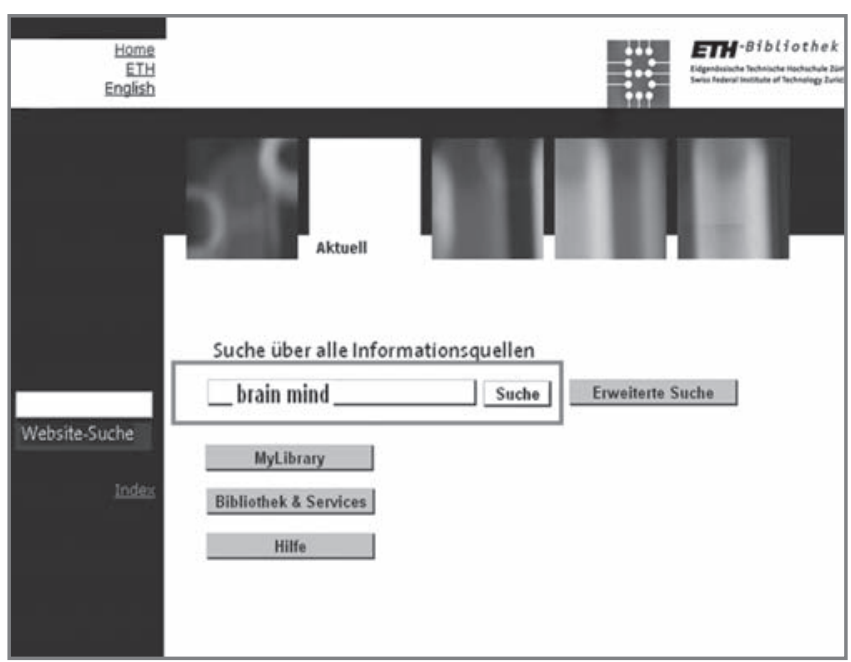

Abb.9: Einstiegsseite in das „Wissensportal ETH Zürich“20 (Recherchebegriff: „brain mind“)
Einsatz von Unternehmensportalen. Whitepaper. Stuttgart 2004 (<www.ebi.iao.fraunhofer.de/Whitepaper\%20 Was\%20ist\%20ein\%20Portal_mit\%20Logo.pdf>).

McGeary, Tim: MyLibrary. The library's response to the campus portal. In: Online Information Review 29 (2005) No. 4, pp. 365-373.

Morgan, Eric L.: MyLibrary in your library could make for satisfied patrons. In: Computers in Libraries 18 (1998) No. 5, p. 40.

Morgan, Eric L.: Putting the „my“ in MyLibrary. In: Library Journal 128 (2003) 17, p. 24-26.

Müller, Ursula: MyLibrary@ETH: Personalisierter Zugang zu elektronischen Informationsressourcen. In: Auf dem Weg zur digitalen Bibliothek. Zürich 2005, S. 137-152.

Neubauer, Wolfram: Die notwendigen Informationsressourcen zur richtigen Zeit am richtigen Ort. Das Portal

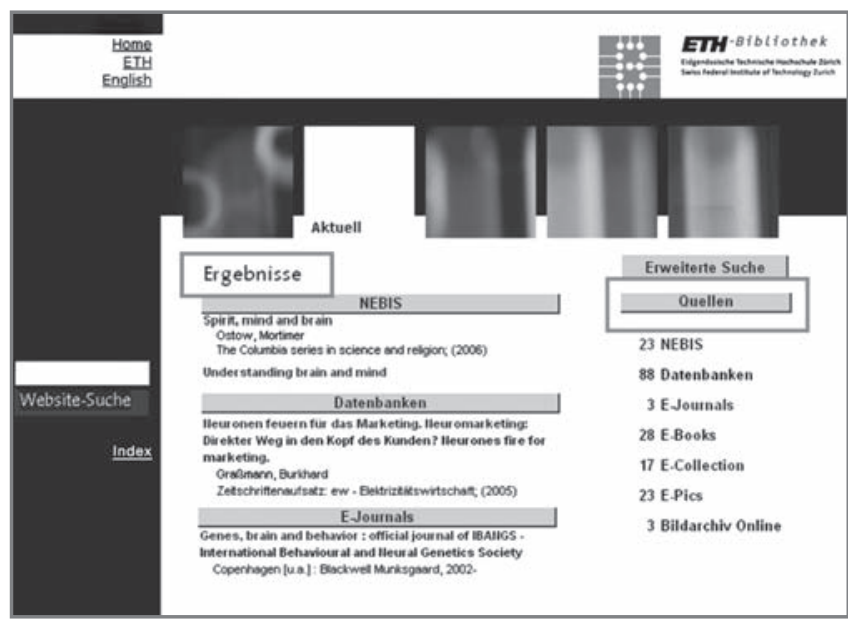

Abb10: Ergebnis der Recherche nach „brain mind“

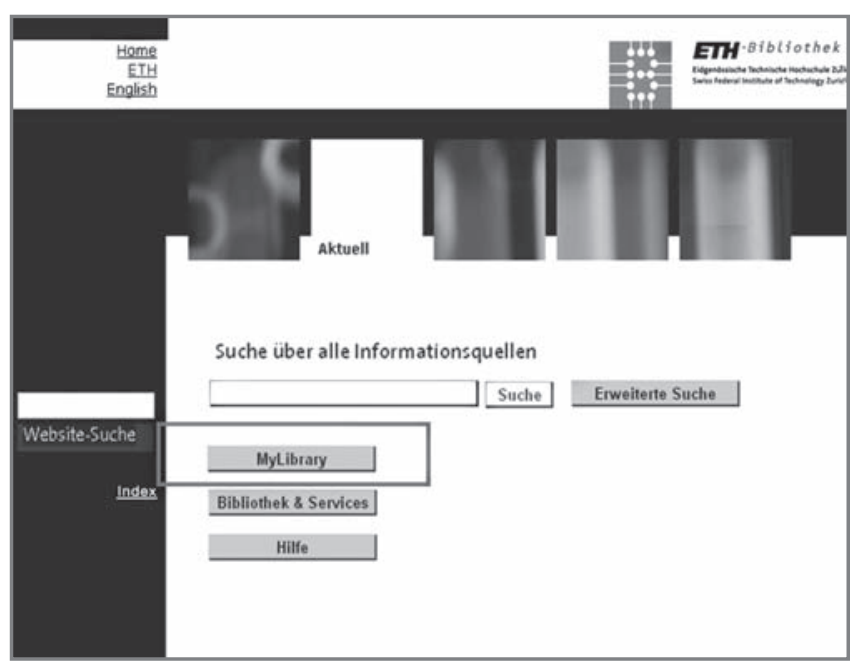

Abb.11: Rechercheeinstieg über „myLibrary“

20 Um eine Vorstellung über die Idee zu gewinnen, wurde die alte (gegenwärtige) Version der Homepage der ETH-Bibliothek verwendet. 


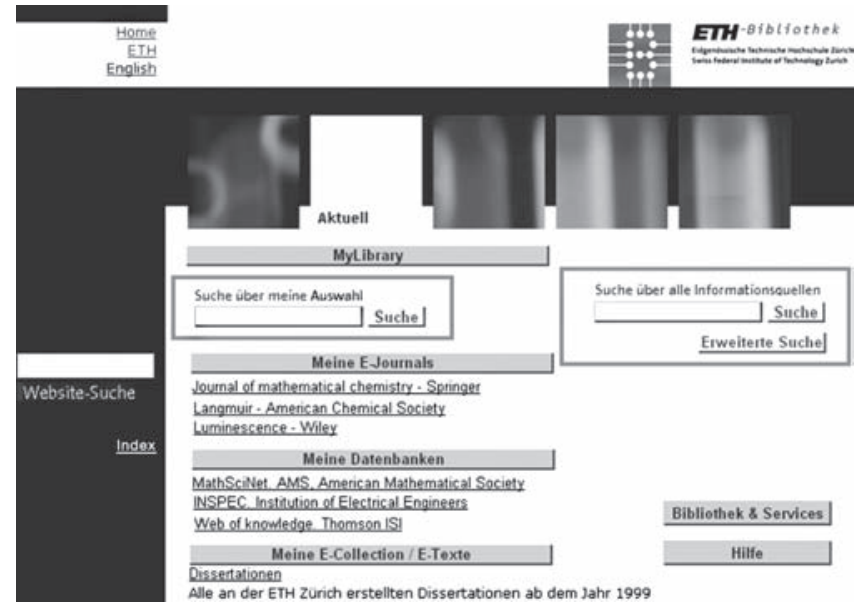

Abb.12: Mögliche Darstellung des Suchergebnisses
myETH als Informationshub der ETH Zürich. In: B.I.T. online 8 (2005) Nr. 2, S.115-122.

Pace, Andrew K.: Should MyLibrary be in your library? In: Computers in Libraries 21 (2001) No. 2, p. 49.

Portals: Frequently asked questions / Ed. by The Joint Information Systems Committee. 2003 (<www.jisc.ac.uk/ index.cfm?name=ie_portalsfaq $>$ ).

\section{Anschrift des Autors:}

Dr. Wolfram Neubauer

ETH Zürich, ETH-Bibliothek

HG J 31.3

Rämistrasse 101

$\mathrm{CH}-8092$ Zürich

Tel: +41 446322125

Fax: +41446321357

E-Mail: neubauer@library.ethz.ch 